\title{
Gestión Pedagógica en la Disciplina Contabilidad Financiera
}

\author{
Cynthia Mabel Ramírez González \\ cynthiamabelramirezgonzalez@gmail.com
}

Universidad Nacional de Pilar

\section{RESUMEN}

El artículo tiene como objetivo describir la gestión pedagógica en la disciplina Contabilidad Financiera utilizada por los docentes de la Facultad de Ciencias Contables Administrativas y Económicas de la Universidad Nacional de Pilar en el año 2019. Se analizó desde tres dimensiones, los cuales fueron metodología de enseñanza, recursos didácticos y competencia docente. La investigación adoptó un enfoque cuantitativo, con diseño observacional, transversal, prospectivo y según el nivel de análisis fue descriptivo, como técnica de recolección de datos se realizó un censo, aplicando un cuestionario a cinco docentes de la disciplina. Los hallazgos principales fueron la utilización de planeamiento didáctico, estructura de los momentos didácticos, estudio de casos, y en menor proporción el uso de los recursos tecnológicos pertinentes al área, sin embargo, los docentes consideran importante la utilización de los mismos, realizan capacitaciones y actualizaciones tanto en el área pedagógico como profesional y gestiones para la implementación de recursos tecnológicos en el área contable. Así también, el 80\% de los docentes apuestan a la adquisición de los mismos a través de la autogestión.

Palabras clave: Gestión pedagógica; contabilidad financiera; metodología de enseñanza; recursos didácticos; competencia docente. 


\title{
Pedagogical Management in the Financial Accounting Discipline
}

\begin{abstract}
The article aims to describe the pedagogical management in the Financial Accounting discipline used by the teachers of the Faculty of Administrative and Economic Accounting Sciences of the National University of Pilar in 2019. It was analyzed from three dimensions, which were teaching methodology, teaching resources and teaching competence. The research adopted a quantitative approach, with an observational, cross-sectional, prospective design and according to the level of analysis it was descriptive, as a data collection technique a census was carried out, applying a questionnaire to five teachers of the discipline. The main findings were the use of didactic planning, structure of didactic moments, case studies, and to a lesser extent the use of technological resources relevant to the area, however, teachers consider their use important, carry out training and updates both in the pedagogical and professional areas and procedures for the implementation of technological resources in the accounting area. Likewise, $80 \%$ of teachers bet on the acquisition of them through self-management.
\end{abstract}

Keywords: Pedagogical management; financial Accounting; teaching methodology; didactic resources; teaching competence.

Artículo recibido: 07. julio. 2020 Aceptado para publicación: 12. setiembre. 2020

Correspondencia: cynthiamabelramirezgonzalez@gmail.com Conflictos de Interés: Ninguna que declarar 


\section{INTRODUCCIÓN}

La Universidad, participa del sistema educativo, formando profesionales competentes en condiciones de enfrentar los desafios de la sociedad actual, en un mundo laboral de crecientes exigencias (De Castro, Closas, \& Cruz, 2019, pág. 66).

Por ello debe ofrecer medios esenciales que favorezcan la formación de sus estudiantes con las exigencias del actual mercado laboral.

Esto implica considerar los recursos con los que cuentan y si los mismos son suficientes y actualizados para brindar a los alumnos educación de calidad.

Por lo tanto, Rico (2016) indica que los docentes y directivos deben asumir nuevos roles con la capacidad de actuar de manera autónoma y tomar decisiones que les permitan acceder al mundo globalizado de la educación y de la sociedad, gestionando procesos adecuados de acuerdo a la realidad social.

En este sentido, Díaz (2019) menciona sobre la gestion educativa, señalando como un nuevo enfoque de actitud positiva hacia el cambio que se desarrolla de manera crítica y constante, de modo a anticiparse a situaciones futuras.

Lopez y Hauman (2018) indican que existe una relación significativa entre la gestión de la calidad y la satisfaccion del estudiante por lo que conlleva a considerar de manera particular el reconocimiento de las necesidades y expectativas de estudiantes, y las demandas de los mercados laborales.

A la gestión educativa se la relaciona con un conjunto de acciones que se dan en el interior de una institución educativa, integrando factores personales, pedagógicos e institucionales que conllevan al logro de los objetivos (Ministerio de Educación como se citó en Márquez Paz, 2018).

En este contexto uno de los factores de la gestión educativa es la pedagógica, la cual se realiza en el aula para fortalecer el proceso de enseñanza como el de aprendizaje, hace énfasis en la planificación y en la importancia de utilizar los medios tecnológicos, pedagógicos e investigativos para alcanzar los resultados de aprendizaje. 
La gestión pedagógica incumbe prácticamente la labor docente, teniendo en cuenta los aprendizajes esperados en los estudiantes; el enfoque del proceso de enseñanzaaprendizaje; las estrategias metodológicas y didácticas, la evaluación de aprendizajes y el uso de materiales y recursos didácticos (SFDMA, 2014, como se citó en Uribe López, Arredondo Cortés, \& López Mayoral, 2018).

Rodríguez (2000) menciona que la gestión pedagogica son los procesos de interacción en el aula: explicaciones, contenidos, recursos, actividades, disciplina, y uso del tiempo.

Por otro lado, la gestión pedagógica se encarga de planificar, evaluar y certificar, el desarrollo de prácticas pedagógicas, actualización y desarrollo personal y profesional de docente (MINEDU 2016, como se citó en Márquez Paz, 2018).

De este modo se puede definir que la gestión pedagógica es la manera de planificar y organizar la enseñanza, utilizando estrategias metodológicas, el uso de materiales y recursos didácticos, teniendo de base la competencia docente en su formación personal como profesional.

Por tal motivo, es importante "el liderazgo del profesor como un objetivo clave de la gestión en los centros educativos" (Martínez de Soria \& Ibarrola García, 2015, pág. 55).

En este sentido es relevante que la Universidad a través de sus docentes practiquen la gestión pedagógica desde la disciplina que les incumbe desarrollar, buscando siempre mejorar el proceso de enseñanza y que los alumnos de esta manera sean beneficiados.

Por ello, en este artículo se toma en cuenta la gestión pedagógica en la disciplina Contabilidad Financiera, dentro de la carrera Contaduría ya que es una materia que está expuesta a los cambios sociales y laborales, por los cambios vertiginosos de la tecnología.

Por lo tanto, es necesario propiciar el uso de estrategias de enseñanza propias del área contable de acuerdo a la realidad que viven las empresas ya que la enseñanza de la disciplina, tradicionalmente se ha visto calificada por la utilización de metodología pobre, fundamentada en la repetición de registros contables de acuerdo a una guía o conocimientos del docente (López Jara \& Cañizares Roig, 2018, pág. 12). 
Por lo que actualmente los registros contables manuales están siendo reemplazadas por registraciones electrónicas, de esta manera se apunta a individuos con capacidad de decisión, asesor y con sólidos conocimientos en normas contables (Ugalde, 1999, como se citó en Labarca Márquez, Navarro Reyes, \& Suárez Capote, 2013).

Siguiendo con esta línea Pérez Sánchez y Ávalos Díaz (2018) mencionan que "La contabilidad electrónica es el nuevo lenguaje del contador público, todo lo que antes se hacía en formatos de papel, hoy cambió gracias a la demanda de la tecnología” (p.63).

Por tal motivo es significativo mencionar la importancia que tienen los estudiantes en poseer conocimientos necesarios de la contabilidad electrónica para poder desenvolverse en un futuro en el ámbito laboral, haciendo actividades prácticas para su mayor aprendizaje (Pérez Sánchez \& Ávalos Díaz, 2018).

Por consiguiente, se considera a la Gestión pedagógica docente de vital importancia, partiendo siempre de que teniendo una buena preparación tanto en el área profesional como pedagógico podrá utilizar los recursos didácticos necesarios y metodológicos, propios de la disciplina contabilidad Financiera, brindando a sus alumnos conocimientos pertinentes y actuales para el desempeño efectivo en el mercado laboral, de ahí es importante la autogestión como punto de partida para cumplir con la misión docente el cual es desarrollar el aprendizaje significativo en sus alumnos.

Por lo tanto, el objetivo de este artículo es describir la gestión pedagógica de los docentes en la disciplina Contabilidad Financiera de la Facultad de Ciencias Contables, Administrativas y Económicas de la Universidad Nacional de Pilar, teniendo en cuenta que de eso depende la adquisición de competencias que se ajustan a la realidad del mercado laboral.

En este sentido se toma en cuenta los siguientes respaldos teóricos que hacen referencia al tema abordado.

\section{Metodologías de Enseñanza}

Hamed, Rivero, \& Martín del Pozo, (2016) mencionan que la metodología es un ámbito curricular esencial ya que compone la principal preocupación de los docentes cuando 
tienen que enseñar un contenido de ciencias, se lo relaciona principalmente con las actividades, constituyéndose el elemento central, ya que pueden apreciarse las interacciones didácticas, la finalidad que tienen, los contenidos, lo que hace el profesor y los estudiantes, la distribución del tiempo y del espacio, los recursos que se utilizan, etc.

El primer factor de la metodología de enseñanza es la planeación didáctica.

En este sentido, "la planeación didáctica implica la organización de un conjunto de ideas y actividades que permiten desarrollar un proceso educativo con sentido, significado y continuidad" (Ascencio Peralta, 2016, pág. 109).

Por ello, para la organización de una clase se debe tomar en cuenta la estructura que las componen, como el inicio, desarrollo y cierre, ya que de esa manera se logra la secuencia pedagógica.

Para un docente de formación profesional, el proceso de enseñanza-aprendizaje se orienta a través de la siguiente metodología: inicia con un diagnóstico, continúa con la presentación del contenido científico y la exposición, hasta llegar a la evaluación de los conocimientos dirigidos ( López Jara \& Cañizares Roig, 2018, pág. 8).

"El manejo de los tiempos en la sesión ayuda al docente a planear mayor variedad de actividades y a controlar el tiempo determinado para cada una, mejorando la secuencia y estructura de la clase" (Ascencio Peralta, 2016, pág. 124).

Planificar los encuentros respetando los momentos de la clase, utilizando en cada uno de ellos las estrategias pertinentes (Montilla P A. I., 2016).

En ese contexto, la planificación de una clase debe estar situados en distintos momentos a modo de organizarla puede responder al esquema clásico: inicio, desarrollo y cierre.

Según el Ministerio de Educación y Cultura (2016) menciona que en el inicio se toma los conocimientos previos de los alumnos para relacionarlos con los nuevos, utilizando la motivación, lluvias de ideas, videos de corta duración, para el desarrollo se utiliza técnicas claves para cada materia identificando aspectos más importantes del contenido 
desarrollado, realizar inferencias, analizar y resolver situaciones problemáticas, consultar fuentes bibliográficas, realizar actividades colaborativas grupales e individuales contextualizadas, dando autonomía a los educandos, y para el cierre se sugiere reflexión sobre lo aprendido, a través del resumen e interpretación de ideas principales, el intercambio de opiniones, la elaboración de respuestas personales, la comprobación de ideas, la formulación de nuevas preguntas, socialización de los resultados.

Considerando la estructura de una clase, la cual rige para todas las áreas, se toma en cuenta la materia en cuestión describiendo la metodología utilizada.

En la enseñanza de la Contabilidad se hace necesario incorporar el uso de simuladores en cada área curricular, manejo de plataformas virtuales de apoyo a la docencia, apoyos de herramientas multimedios; consulta de bibliotecas digitales, consultas de bases de datos científicas; y en general materiales didácticos motivantes hacia los alumnos, a través de los diferentes tipos de comunicaciones (Ruiz, Martinez, \& Sánchez, 2015, pág. 40).

La metodología debe estar sustentada en la utilización de didácticas creativas las cuales deben estar acompañadas por la experiencia es decir deben tener un contexto real que muestre la aplicación eficiente que tienen los conocimientos al ponerlos en práctica en la vida real ( Cassiani \& Zabaleta, 2016).

La enseñanza de la Contabilidad requiere preparación, metodología y creatividad del docente para combinar estos elementos y promover un aprendizaje significativo en el estudiante. En la medida en que el conocimiento científico es presentado de forma atractiva, incrementará el interés del estudiante por profundizar su análisis y, por tanto, la disposición de este para aprender (López Jara \& Cañizares Roig, 2018, pág. 7).

Las estrategias de enseñanza en la materia Contabilidad pueden ser diseñadas de tal manera que estimulen a los estudiantes a observar, analizar, opinar, formular hipótesis, buscar soluciones y descubrir el conocimiento por sí mismos o procurar la transferencia de información o conocimiento (Montilla, 2016). 
El futuro de la formación contable depende en gran medida de los cambios que los docentes estén dispuestos a hacer para incorporar en el proceso de aprendizaje los elementos existentes en el mundo actual globalizado y tecnológico, para que el modelo de enseñanza tradicional se adecue a las necesidades del mercado laboral de los profesionales de la contaduría pública (Labarca Márquez, Navarro Reyes, \& Suárez Capote, 2013)

Es necesaria la combinación de varios métodos, como el expositivo, método de lectura dirigida, enseñanza mediante módulos, método de solución de casos, método del panel, método de la entrevista, entre otros (Nerici, 1985, como ce citó en López Jara \& Cañizares Roig, 2018).

Montilla (2016) realiza algunas recomendaciones didácticas tales como:

Activar y generar conocimientos previos mediante la motivación y la presentación de objetivos y el uso de diferentes tipos de estrategias de enseñanza, como la lluvia de ideas, ilustraciones, el aprendizaje basado en problemas (ABP), entre otras, logrando el interés y participación del grupo, hacer uso de estrategias innovadoras como los mapas conceptuales y las redes semánticas, como una forma de codificar visual y semánticamente los conceptos o conocimientos, propugnar por una enseñanza a través de la reflexión en la acción, por tanto, la formación docente debe continuarse en tres planos: conceptual, reflexivo y práctico; y de la misma forma debe orientarse hacia el estudiante de educación superior y de otros niveles, utilizando técnicas grupales y dinámicas de grupos en cada objetivo.

Por otro lado, Cassiani y Zabaleta (2016) señalan las siguientes estrategias para el área de Contabilidad

Aprendizaje cooperativo los alumnos trabajan divididos en pequeños grupos, y son evaluadas según la productividad del grupo.

Aprendizaje basado en problemas (ABP) los estudiantes aprenden en pequeños grupos, partiendo de un problema, a buscar la información que necesita para comprender el problema y obtener una solución, bajo la supervisión de un tutor 
Exposición/Lección magistral: Presentar de manera organizada información (profesor-alumnos; alumnos-alumnos). Activar la motivación y procesos cognitivos.

Estudio de casos: los alumnos analizan situaciones profesionales presentadas por el profesor, con el fin de llegar a una conceptualización experiencial y realizar una búsqueda de soluciones eficaces.

Simulación y juego: Dan a los estudiantes un marco donde aprender de manera interactiva por medio de una experiencia viva.

Un cambio de método se haya justiciado, más aún, debido al avance de la sociedad que pone a nuestra disposición herramientas, como los medios informáticos, que facilitan el aprendizaje interactivo. Además, las empresas demandan profesionales capaces de adaptarse al entorno, con capacidad de análisis crítico y motivación para seguir aprendiendo ( Cassiani \& Zabaleta, 2016).

Entre las estrategias que el docente podría optar para el desarrollo de la clase, sugeridas por el estudiante, se encuentran el debate académico, los concursos de conocimientos y las exposiciones de contenidos investigados, principalmente. (López Jara \& Cañizares Roig, 2018, pág. 9).

La facultad debe establecer alianzas estratégicas con los proveedores de software contables para el otorgamiento de licencias de uso con carácter educativo, a fin de que los estudiantes de las asignaturas contables realicen las prácticas en un sistema automatizado de uso común en las empresas (Labarca Márquez, Navarro Reyes, \& Suárez Capote, 2013, pág. 399).

Continuando con los elementos que se hacen necesario para las estrategias de enseñanzas son:

\section{Recursos Didácticos}

Los recursos didácticos son entendidos como los medios empleados por el docente para fortalecer el proceso educativo, como las guías, los libros, la pizarra, los videos, entre otros (López Jara \& Cañizares Roig, 2018, pág. 9). 
Sin embargo la innovación en la pedagogía está basada hoy en día en las herramientas de las tecnologías de la información y comunicación (TIC) (Novillo Maldonado, Espinosa Galarza, \& Guerrero Jirón, 2017).

La innovación es un cambio que alcanza a nuestro entorno diariamente, la tecnología es la principal causante de que esto suceda a través de nuevas invenciones, métodos y técnicas, claramente, la contabilidad no está exenta y actualmente, el modo de contabilizar y las leyes fiscales se mantienen en constante cambio, adaptándose a las tecnologías habientes (Pérez Sánchez \& Ávalos Díaz, 2018, pág. 248).

La educación superior debe sacar provecho de los avances logrados por la tecnología de la información, que facilitan enormemente la recopilación y el análisis de los datos (Unesco 2000 como se citó en Cassiani \& Zabaleta, 2016).

Dentro de las herramientas TIC más usadas, se pudo concluir que las herramientas TIC más usadas en los procesos de enseñanza son: las Redes sociales, Correo Web, Software de presentación, (Novillo Maldonado, Espinosa Galarza, \& Guerrero Jirón, 2017).

Es necesario incorporar las TICs para lograr la armonización entre la Universidad y el mercado laboral, por lo que la enseñanza en la práctica contable debe hacerse a través del manejo de software o sistemas contables (Labarca Márquez, Navarro Reyes, \& Suárez Capote, 2013).

En tal sentido se hace hincapié a la importancia que tiene la contabilidad electrónica como una nueva y obligatoria modalidad de contabilizar, resaltando la interrelación que hay entre educación y tecnología, formándose día con día como parte de una nueva cultura académica (Pérez Sánchez \& Ávalos Díaz, 2018, pág. 247).

Por tal motivo, el uso de las Tic en el área contable es de suma importancia ya que el empleo del software permite la realización de casos con datos contables y tributarios reales, lo cual facilita el trabajo en grupos guiados por el docente (Benítez Median \& Jiménez, 2017).

Se hace necesario e importante la utilización de un software contable como estrategia de enseñanza-aprendizaje pues permite que el egresado en contabilidad pueda contar con 
competencias en el manejo de programas contables y por consiguiente facilitar su inserción en el campo laboral (Vizcaino \& Becerra, 2019).

Existen actualmente universidades que han incorporado a la Licenciatura de Contaduría Pública diversas modalidades de educación a distancia adoptando el uso de las TICs, tal es el caso de la Universidad Autónoma del Carmen del Estado de México y la Universidad Pública del Departamento del Valle-Colombia. Los estudiantes de esta Licenciatura realizan prácticas en software contables, utilizan simuladores de negocios, plataformas educativas, juegos interactivos, entre otras, lo que ha permitido fortalecer su desarrollo profesional (Labarca Márquez, Navarro Reyes, \& Suárez Capote, 2013).

Ruiz, Martinez y Sánchez, ( 2015) mencionan que:

En la enseñanza de la contaduría y administración es necesario incorporar el uso de simuladores en cada área curricular, manejo de plataformas virtuales de apoyo a la docencia, apoyos de herramientas multimedios; consulta de bibliotecas digitales, consultas de bases de datos científicas; y en general materiales didácticos motivantes hacia los alumnos (p.40).

Por consiguiente, el estudiante debe adquirir los conocimientos teórico-prácticos necesarios mediante el uso de todas las herramientas que proporciona la informática, con el objetivo de integrarse a la nueva sociedad (Teruel Mulet, Fernández Flores, \& Martinez Dominguez, 2015).

Teniendo en cuenta todo lo mencionado, los mismos estudiantes "sugieren la utilización de un programa contable en complemento a la enseñanza debido a que, en la actualidad, el registro de los hechos económicos es realizado en softwares contables y no en libros, como se practica en las clases” (López Jara \& Cañizares Roig, 2018, pág. 9).

Según Ruiz y Sánchez (2015) la institución a traves de sus docentes debe contar con una visión de apartetura al uso de la tecnologia, y buscar recursos para el equipamiento e infraestructura tecnologica, implemtar programa permanente de desarrollo personal docente en relacion al uso de la tecnologia. 
Por tal razón es importante la utilización de softwares contables como complemento en el proceso pedagógico ya que el actual mercado laboral exige profesionales con conocimientos pertinentes del área y uno de ellos es la utilización de las Tics.

\section{Competencia Docente}

Zabalza (2007) menciona que la competencia docente se relaciona al deber ser y el hacer de los profesores formando estudiantes en competencias profesionales valiosas, considerando un conjunto de conocimientos, habilidades cognitivas, actuaciones prácticas de actitudes, formas de actuación, sensibilidades, valores.

Habilidad principal, en tanto que define la identidad del profesor desde una visión holística de su ejercicio profesional (Korthagen como se citó en Cateñada, Esteve, \& Adell, 2018, pág. 10).

Según Hernández, Recalde y Luna (2015) es transcendental tener en cuenta las competencias que los docentes deben contar, para desarrollar con éxito su función en la orientación del logro de las competencias de los estudiantes; por sobre todo, cuando tienen la responsabilidad de formar por competencias estudiantes para el mundo laboral.

Las competencias docentes descritas son: "Planificar, Selección de contenidos, Comunicar, Manejo de Tecnología de la Información y Comunicación, Diseño de Metodología, Relación con el estudiante, Tutorizar, Evaluar, Reflexionar e Identificación" (Goset Poblete \& Navarrete Ponce, 2017).

De este modo, para que la Universidad pueda cumplir sus tareas académicas, laborales e investigativas requiere de profesores preparados, que no sólo sepan el contenido científico, sino que sepan enseñar lo que necesita la sociedad, de aquí la necesidad de que en la universidad se enseñe a los profesores a educar, para que los estudiantes aprendan a aprender (Caceres, 2019).

La contabilidad como cualquier otro proceso, está en constante cambio con el ambiente en el que lo envuelve, los educadores lo deben tener en cuenta al momento de impartir cátedra, para instruir a la nueva generación de profesionistas que se avecinan en pos de ellos (Pérez Sánchez \& Ávalos Díaz, 2018, pág. 263). 
Por esta razón en el proceso de formación profesional, el docente contable tiene un rol fundamental, pues es quién debería poseer la capacidad de expandir sus saberes a un grupo de estudiantes para el apropiado ejercicio de la profesión contable (Cardona Restrepo, Varón Gómez, Bonilla Solórzano, \& Rincón Soto, 2016).

En este sentido es fundamental que los docentes se actualicen y capaciten, para contribuir con la formación del estudiante, lo que es de suma importancia para su formación debido a las estrategias implementadas, lo que es un beneficio para que el alumno tenga un buen desempeño y sea un buen profesional ( Cassiani \& Zabaleta, 2016).

Por ello los docentes de contaduría de las universidades deberán contar con las capacidades siguientes: conocer métodos didácticos específicos de la Contabilidad Electrónica, la competencia tecnológica práctica y discernir el acompañamiento adecuado a los alumnos en el proceso formativo de la Contabilidad, en medios electrónicos (Lee Pérez, 2017).

De acuerdo a esto, es importante resaltar que el contador debe ser un profesional analítico, crítico y con espíritu investigativo que se actualice sobre las nuevas normas y técnicas, ya que al momento de llevarlas a la práctica le sirven de apoyo para resolver problemas presentes en su diario vivir, contribuyendo con el desarrollo de la comunidad en general ( Cassiani \& Zabaleta, 2016).

En este sentido es necesario formar al docente, tanto en el manejo de las TIC, como en la formulación de estrategias pedagógicas, que permitan el uso vinculante de las tecnologías en el tiempo presencial y no presencial ( Cassiani \& Zabaleta, 2016).

Por eso importante la actualización permanente de los docentes en nuevas estrategias orientadas hacia el aprendizaje significativo en beneficio de los estudiantes (Montilla, 2016).

Rico (2016) comenta que Influyen en el rendimiento de los estudiantes que exista docentes especializados y con experiencia, haciéndose necesario que los actores del ámbito educativo gestionen procesos adecuados y coherentes con la movilidad social que acontece en la sociedad. 
Por consiguiente en el proceso educativo los gestores de la educación son los responsables de asegurar que se mejoren los resultados de aprendizaje de los estudiantes (Saltos Solís, 2015, pág. 57).

Siguiendo con esta linea Martínez y Ibarrola (2015) consideran el liderazgo del profesor como uno de los principales cometidos en la gestión de los centros educativos.

En resumen, el docente universitario debe estar abocado al profesionalismo constante ya que vivimos en una era de cambios vertiginosos y es de vital importancia la actualización y conocimientos de las nuevas implicancias que corresponden al área educativa y profesional, debe tener capacidad de gestión para alcanzar los recursos deseados en el proceso pedagógico.

De esta manera ofrecerá a los estudiantes soluciones pertinentes a los problemas planteados utilizando las adecuadas metodologías de enseñanza.

\section{MATERIALES Y MÉTODOS}

La investigación adoptó un enfoque cuantitativo, observacional ya que no existe intervención de la investigadora sobre las variables, transversal puesto que las variables son medidas en una sola ocasión, prospectivo porque los datos fueron recogidos por fuente primaria, y según el tipo de análisis fue descriptivo ya que describe parámetros de la población. Se recurrió al censo como técnica de recolección de datos, mediante la aplicación de un cuestionario como instrumento documental, previamente realizando una prueba piloto a la población, conformado por cinco docentes de la disciplina Contabilidad Financiera, de la carrera Contaduría Pública Nacional de la Facultad de Ciencias Contables, Administrativas y Económicas de la Universidad Nacional de Pilar (UNP) año 2019.

El cuestionario tuvo seis ítems, en los dos primeros se utilizó la escala de Likert compuesta del 1 al 4, donde el 4 fue alto, el 3 Medio, el 2 bajo y el 1 no aplica, teniendo en cuenta la utilización de la metodología de enseñanza y recursos didácticos, en los tres restantes se utilizó el cuestionario cerrado. 


\section{RESULTADOS Y DISCUSIÓN}

Los principales resultados de esta investigación señalan que los docentes practican una estructura pedagógica en su proceso didáctico como el inicio desarrollo y cierre, utilizan diversas técnicas metodológicas del área contable, en tanto a los recursos didácticos en un alto porcentaje se manejan con medios tradicionales y no tecnológicos sin embargo el $80 \%$ de los docentes consideran oportuno la implementación de este último, por lo que los mismos realizan gestiones para la implementación de este recurso.

Los docentes en su totalidad realizan capacitaciones en el área profesional como metodológica por parte de la Facultad de Ciencias Contables, Administrativas y Económicas (FCCAYE) y por gestión propia.

Teniendo en cuenta la información proporcionada se detalla a continuación resultados más específicos a través de la utilización de tablas.

Primeramente, se presentan los resultados teniendo en cuenta la escala de Likert.

Tabla n 1: Metodología de enseñanza.

\begin{tabular}{|c|c|c|c|c|c|c|c|c|c|c|c|c|c|c|}
\hline & \multicolumn{2}{|c|}{$\begin{array}{c}\text { Planeamiento } \\
\text { Didácticos }\end{array}$} & \multicolumn{2}{|c|}{$\mathrm{ABP}$} & \multicolumn{2}{|c|}{$\begin{array}{c}\text { Estudio de } \\
\text { Casos }\end{array}$} & \multicolumn{2}{|c|}{$\begin{array}{l}\text { Aprendizaje } \\
\text { Cooperativo }\end{array}$} & \multicolumn{2}{|c|}{ Simulación } & \multicolumn{2}{|c|}{$\begin{array}{c}\text { Socialización } \\
\text { de los } \\
\text { Resultados. }\end{array}$} & \multicolumn{2}{|c|}{ Retroalimentación } \\
\hline & $\mathrm{Fr}$ & $\%$ & $\mathrm{Fr}$ & $\%$ & $\mathrm{Fr}$ & $\%$ & $\mathrm{Fr}$ & $\%$ & $\mathrm{Fr}$ & $\%$ & $\mathrm{Fr}$ & $\%$ & Fr. & $\%$ \\
\hline Alto & 5 & 100 & 3 & 60 & 5 & 100 & 3 & 60 & 1 & 20 & 5 & 100 & 2 & 40 \\
\hline Medio & & & 2 & 40 & & & 2 & 40 & 1 & 20 & & & 3 & 60 \\
\hline Bajo & & & & & & & & & 2 & 40 & & & & \\
\hline $\begin{array}{l}\text { No } \\
\text { aplica }\end{array}$ & & & & & & & & & 1 & 20 & & & & \\
\hline Total & 5 & 100 & 5 & 100 & 5 & 100 & 5 & 100 & 5 & 100 & 5 & 100 & 5 & 100 \\
\hline
\end{tabular}

Fuente: Elaboración propia. 
El $100 \%$ de los docentes realizan planeamientos didácticos incluyendo en su estructura los momentos didácticos como lo dice Ascencio (2016) utilizar la planeación didáctica permite la organización de un conjunto de ideas y actividades las cuales conlleva a desarrollar el proceso pedagógico con sentido y pertinencia, en concordancia a lo que menciona Montilla (2016) es importante planificar los encuentros respetando los momentos de la clase, utilizando en cada uno de ellos las estrategias pertinentes.

La técnica que mayoritariamente utilizan los docentes es el estudio de casos en un $100 \%$ seguidamente por el aprendizaje basado en problemas y aprendizaje cooperativo, por último y con menor porcentaje utilizan la simulación.

Asencio (2016) por su parte menciona que el aprendizaje colaborativo propicia el aprendizaje significativo con mayor participación y reflexión.

Por otro lado Ruiz, Martinez, \& Sánchez, ( 2015) comenta que en la enseñanza de la contaduría es necesario incorporar el uso de simuladores.

Para las actividades de cierre según el Ministerio de Educación y Cultura (2016) mencionan que las preguntas aleatorias y socialización de resultado, es un mecanismo de control para que se compruebe el grado de aprendizaje del alumno, en sentido el $100 \%$ de los docentes indicaron que utilizan en frecuencia alta la socialización de resultados, sin embargo, la retroalimentación es utilizado solo en un $40 \%$.

Por último, Ascencio ( 2016) menciona que se debe realizar una retroalimentación continua que permita al estudiante a observar aspectos positvos como negativo de los aprendizajes adquridos. 
Tabla $\mathbf{n}^{\circ}$ 2: Recursos didácticos

\begin{tabular}{|l|l|l|l|l|l|l|}
\hline & \multicolumn{2}{|c|}{$\begin{array}{r}\text { Registros } \\
\text { manuales }\end{array}$} & \multicolumn{2}{r|}{$\begin{array}{r}\text { Software } \\
\text { contable }\end{array}$} & \multicolumn{2}{c|}{ Plataformas Virtuales } \\
\hline & Fr. & $\%$ & Fr. & $\%$ & Fr. & $\%$ \\
\hline Alto & 5 & 100 & & & & \\
\hline Medio & & & 1 & 20 & 3 & 60 \\
\hline Bajo & & & 2 & 40 & 2 & 40 \\
\hline No aplica & & & 2 & 40 & & 100 \\
\hline Total & 5 & 100 & 5 & 100 & 5 & \\
\hline
\end{tabular}

Fuente: Elaboración propia

Prevalece los registros contables manuales en un $100 \%$ con frecuencia alta, en este sentido Ruiz y Sánchez (2015) mencionan que la institución a través de sus docentes debe contar con una visión de apartetura al uso de la tecnologia, y buscar recursos para el equipamiento e infraestructura tecnologica, por lo que conllevaría a realizar acciones pertinentes en busca a la gestión de recursos actuales en contexto con el mercado laboral teniendo en cuenta los recursos didácticos tecnológicos.

Por otro lado, López Jara y Cañizares Roig (2018) sugieren la utilización de un programa contable en complemento a la enseñanza debido a que, en la actualidad, el registro de los hechos económicos es realizado en softwares contables.

Ruiz, Martinez, \& Sánchez (2015) mencionan que en la enseñanza de la Contabilidad se hace necesario incorporar el uso de simuladores, manejo de plataformas virtuales y herramientas multimedios.

Se asume que faltaría mejorar el uso de las Tic específicamente en las registraciones contables como complemento a los recursos tradicionales, como lo dice Labarca, 
Navarro y Suárez (2013) la formación contable depende en gran medida de los cambios que los docentes realicen para integrar en el proceso pedagógico los recursos actuales tecnológicos que el mercado laboral exige a los profesionales de la Contaduría.

Tabla 3: Software Contable

\begin{tabular}{|c|c|c|c|c|}
\hline & \multicolumn{2}{|c|}{$\begin{array}{c}\text { Importancia del Software } \\
\text { Contable }\end{array}$} & \multicolumn{2}{c|}{ Propuesta de implementación } \\
\hline & Fr. & $\%$ & 4 & 80 \\
\hline $\mathrm{Si}$ & 4 & 80 & 1 & 20 \\
\hline No & 1 & 20 & 5 & 100 \\
\hline Total & 5 & 100 & & \\
\hline
\end{tabular}

Fuente: Elaboración propia.

En este apartado el $80 \%$ de los docentes consideran importante la implementación del software contable porque se hace necesario estar a la vanguardia de los cambios vertiginosos de la tecnología, de este modo Pérez Sánchez \& Ávalos Díaz (2018) mencionan sobre la importancia que tiene la contabilidad electrónica como una nueva y obligatoria modalidad de contabilizar, destacando la interrelación que hay entre educación y tecnología, considerada como una nueva cultura académica.

Del mismo modo, los mismos han realizado propuestas de implementación de los recursos tecnológicos a la Facultad de Ciencias Contables, Administrativas y Económicas, siguiendo con esta linea Martínez y Ibarrola (2015) consideran el liderazgo del profesor como uno de los principales cometidos en la gestión de los centros educativos, por otro lado, Según Ruiz y Sánchez (2015) mencionan que se hace necesario que los docentes gestionen recursos para el equipamiento e infraestructura tecnologica, como actor clave del proceso pedagogico.

Por otro lado, teniendo en cuenta la respuesta del $20 \%$ se puede considerar según Ruiz, Martinez, y Sánchez (2015) que los docentes se resisten al uso de las tecnologías porque 
creen ser expertos de sus disciplinas y suponen que el uso de los mismos reduciría sus posiciones profesionales, se instruyeron especialmente con libros, y no poseen modelos de cómo enseñar con tecnologías.

Tabla 4: Competencia Docente: Tipos de Capacitaciones

\begin{tabular}{|l|l|l|}
\hline & Frecuencia & Porcentaje \\
\hline Ambos & 5 & 100 \\
\hline Área profesional & 0 & 0 \\
\hline Pedagógica & 0 & 0 \\
\hline Total & 5 & 100 \\
\hline
\end{tabular}

Fuente: Elaboración propia.

El $100 \%$ de los docentes manifestaron que realizan capacitaciones en los diferentes ámbitos del saber, en este sentido Caceres (2019) indica que se requiere de profesores preparados no sólo en el contenido científico, sino en la manera de enseñanza, en este orden se menciona que no es suficiente que el docente imparta el saber disciplinar sino también el saber pedagógico (Núñez, Fajardo y Químbayo como se citó en Portocarrero Merino \& Barrionuevo Torres, 2018).

Tabla 5: Competencia Docente: Origen de las capacitaciones.

\begin{tabular}{|l|l|l|}
\hline & \multicolumn{1}{|c|}{ Frecuencia } & \multicolumn{1}{c|}{ Porcentaje } \\
\hline FCCAYE & 0 & 0 \\
\hline Gestión propia & 0 & 0 \\
\hline Ambos & 5 & 100 \\
\hline Total & 5 & 100 \\
\hline
\end{tabular}

Fuente: Elaboración propia. 
Los datos demuestran que los docentes consiguen capacitarse a través de los cursos ofrecidos por la FCCAYE como también por gestiones propia, en este contexto como lo dice Portocarrero\& Barrionuevo (2018) es necesario disponer de programas de capacitación y actualización docente con el propósito de conseguir metodologías actuales de enseñanza, por otro lado, Zabalza (2007) menciona que ser competente se relacion a la forma de actuaciones prácticas y actitudes que el docente debe realizar para cumplir son sus objetivos pedagógicas.

Resumiendo, se menciona que el $100 \%$ de los docentes realizan diferentes tipos de capacitaciones a través de la autogestión y por parte de la FCCAyE.

\section{CONCLUSIÓN O CONSIDERACIONES FINALES}

La investigación permitió describir la gestión pedagógica que realizan los docentes en cuanto a la metodología de enseñanza, los recursos didácticos y a la competencia docente teniendo en cuenta la capacitación, actualización y acciones para lograr mayores recursos pedagógicos en su práctica docente.

En lo que respecta a la metodología de enseñanza los docentes se organizan utilizando planeamiento didáctico siguiendo la estructura de los momentos didácticos los cuales son inicio, desarrollo y cierre, utilizan variadas actividades para cada momento como motivación para el inicio, utilización de variadas técnicas pertinentes al área contable para el desarrollo del proceso pedagógico como Aprendizaje Basados en problema, estudio de casos, simulaciones, aprendizaje colaborativo e individual.

Para el cierre de los contenidos utilizan preguntas aleatorias como la socialización de los resultados, sin embargo, en un bajo porcentaje utilizan la retroalimentación.

En cuanto a los recursos didácticos en general utilizan los tradicionales al área contable como las registraciones en forma manual, pero considerando que es significativo la implementación del software contable, realizando propuestas para su implementación a la FCCAYE.

Por otro lado, en cuanto a la competencia docente, todos realizan capacitaciones y actualizaciones en aspectos pedagógicos como en el área profesional, y el 80\% de los 
mismos poseen posturas abiertas a la inclusión del software contable en las registraciones, realizando gestiones para su implementación de la misma manera para lo que respecta

Se considera resultados positivos al concluir con este artículo, si bien no se utiliza como se quisiera los recursos tecnológicos del área contable, el 80\% de los docentes apuestan a la adquisición de los mismos a través de la autogestión, ya que de esta manera como lo dice Lopez y Hauman (2018) se logrará la satisfaccion del estudiante considerando sus necesidades y expectativas, y la demanda del mercado laboral.

\section{LISTA DE REFERENCIAS}

Ascencio Peralta, C. (2016). Adecuación de la Planeación Didáctica como Herramienta Docente en un Modelo Universitario orientado al Aprendizaje. Revista Iberoamericana sobre Calidad, Eficacia y Cambio en Educación., 109-130.

Benítez Median, R., \& Jiménez, M. B. (2017). Aprendizajes Interactivo en Estudiantes de Contabilidad de la Carrera de Administración de Empresas. Revista Capic Review. , Volumen 14, 47-56.

Caceres, M. L. (2019). La formación pedagógica de los profesores universitarios. Una propuesta en el proceso de profecionalización docente. Revista Iberoamericana de Educación, 1-14.

Cardona Restrepo, L. M., Varón Gómez, M. C., Bonilla Solórzano, I. T., \& Rincón Soto, C. A. (2016). El "ser docente" en contabilidad. Revista Libre Empresa, 103-123.

Cateñada, L., Esteve, F., \& Adell, J. (2018). ¿Por qué es necesario repensar la competencia docente para el mundo digital? Revista de Educación a Distancia(56), 1-20.

Cassiani , D., \& Zabaleta, M. (2016). Metodologías utilizadas en la enseñanza de contabilidad internacional: una propuesta. Revista Interamericana de Investigación, Educación y Pedagogía, RIIEP. , Vol.9 , 1-16. 
De Castro, I. G., Closas, A. H., \& Cruz, R. (2019). Modelo didáctico para la intervención educativa universitaria,. Revista de la Facultad de Ciencias Económicas, 66-90.

Díaz Torres, J. M. (2019). Revisión y Discusión de las posibilidades cívicas en la docencia universitaria. Innovación, Gestión Educativa y Compromiso Activo. De los procesos de cambio al cambio con sentido, 137-150.

Goset Poblete, J., \& Navarrete Ponce, G. (2017). Variables de personalidad y competencias docentes . Revista Iberoamericana de Educación, 51-66.

Hamed, S., Rivero, A., \& Martín del Pozo, R. (2016). El cambio en las concepciones de los futuros maestros sobre la metodología de. Revista Eureka, 476-492.

Hernández Arteaga, I., Recalde Meneses, J., \& Luna, J. (2015). ESTRATEGIA DIDÁCTICA: UNA COMPETENCIA DOCENTE EN LA FORMACIÓN. Revista Latinoamericana de Estudios Educativos (Colombia), 11(1), 73-94.

Labarca Márquez, S. E., Navarro Reyes, Y., \& Suárez Capote, E. (2013). La tecnología en la formación contable. Un paso hacia el futuro. Revista de Ciencias Sociales (RCS), Vol. XIX(No. 2), 390-414.

Lee Pérez, P. (2017). La importancia de la contabilidad electrónica. Realidad y Reflexión, $8-17$.

Obtenido

de

https://www.lamjol.info/index.php/RyR/article/view/4415/4172

Lopez Ibañez, S. S., \& Hauman Nuñez, J. (2018). Gestión de Calidad y Satisfacción de Necesidades en la Universidad Nacional "Micaela Batidas". Investigación y Postgrado, 193-208.

López Jara, A. A., \& Cañizares Roig, M. (2018). Diagnóstico sobre la metodología para la enseñanza de la Contabilidad en la Universidad Católica de Cuenca, Sede Macas. Revista Cubana de Educación Superior, 1-13.

Márquez Paz, B. I. (2018). Gestión Educativa y su relación con la enseñanza-aprendizaje de los estudiantes del Centro de Educación Básica Alternativa Ricardo Palma de Surquillo. Moquegua, Perú: Universidad José Carlos Mariátegui. 
Martínez de Soria, A. B., \& Ibarrola García, S. (2015). Liderazgo del Profesor: Objetivo Básico de la Gestión Educativa. Revista Iberoamericana de Educación, 55-70.

Ministerio de Educación y Cultura. (2016). Módulo de Formación. Capacitación docente Continua y en Servicio. Paraguay.

Montilla P, A. (2016). Consideraciones sobre las estrategias de enseñanzas más efectivas en la Contabilidad. Revista Científica Electrónica de Ciencias Gerenciales, 2357.

Novillo Maldonado, E. F., Espinosa Galarza, M. O., \& Guerrero Jirón, J. R. (2017). Influencia de las TIC en la educación universitaria, caso Universidad Técnica de Machala. Revista INNOVA Research Journal, Vol 2, No. 3, 69-79.

Paz Viltchez, J. E., López Díaz, K., \& Romero Chinguel, L. C. (2017). Preferencia de Universidad y Carreras con mayor demanda en la Region San Martín. Apuntes Universitarios, 8-22.

Pérez Sánchez, E., \& Ávalos Díaz, A. (2018). Contabilidad Electrónica, Enfoque Esencial para la Educación Contable. Hitos de Ciencias Económico Administrativas, 246266.

Portocarrero Merino, E., \& Barrionuevo Torres, C. (2018). Cultura Pedagógica y Competencias del Docente Universitario desde la percepción del estudiante. Investigación y Postgrado, 34(1), 151.

Rico, A. D. (2016). La gestión educativa: Hacia la optimización de la formación docente en la educación superior en Colombia. Sophia(12), 55-70.

Rodríguez, N. (2000). Gestión Escolar y Calidad en la Enseñanza. Educere, 39-46.

Ruiz, J. A., Martinez, M., \& Sánchez, M. (2015). El impacto de las TICs en la calidad de la educación superior. Revista de Investigación en Ciencias Contables y Administrativas, 28-44. 
Saltos Solís, M. (2015). La eficiente Gestión Educativa en los resultados de aprendizaje de los estudiantes universitarios. Revista Ciencia UNEMI, 8(13), 56-70.

Seltzer, J. C. (2006). Contabilidad y Docencia. Lúmina 07, 154-159.

Teruel Mulet, M., Fernández Flores, E., \& Martinez Dominguez, M. (2015). Estrategia Didáctica con el apoyo de las TIC para el desarrollo de habilidades profesionales en la licenciatura en Contabilidad y Finanzas en el modelo semi presencial. Revista Pedagogía Universitaria, Vol. XX No. 1, 71-81.

Uribe López, A., Arredondo Cortés, S. A., \& López Mayoral, M. A. (2018). Diagnóstico de Gestión Institucional: Un estudio de caso en Educación Básica. Revista de la Facultad de Contaduría y Ciencias Administrativas, 9-22.

Zabalza, M. A. (2007). Competencias docentes del prodesorado universitario. En Calidad y desarrollo profesional (págs. 1-217). Madrid, España: Narcea S.A Ediciones. 